\title{
TITLE: COMPARATIVE ANALYSIS OF PERIPHERAL CORNEAL GEOMETRY IN HEALTH AND KERATOCONUS
}

Running Title: Peripheral corneal angles in health and keratoconus

Authors:

Enric Mas-Aixala, MSc ${ }^{\wedge}$

Joan Gispets, $\mathrm{PhD}^{\wedge}$

Núria Lupón, $\mathrm{PhD}^{\wedge}$

Genís Cardona, $\mathrm{PhD}^{\wedge}$

^ Universitat Politècnica de Catalunya, Faculty of Optics and Optometry, Optics and Optometry Department, c/Violinista Vellsolà, 37, E08222, Terrassa, Spain.

\section{Corresponding Author:}

Joan Gispets

Facultat d'Òptica i Optometria de Terrassa

Violinista Vellsolà, 37

E08222 Terrassa, Catalonia, Spain

E-mail address:

joan.gispets@upc.edu

Phone:

+34937398310

Figures: 6

Tables: 2

Submission Date: $9^{\text {th }}$ of November 2016: Revised $17^{\text {th }}$ of March 2017

Statement of financial disclosure and conflict of interest: none of the authors have any involvement in any organisation or entity with direct financial interest in the subject matter or materials discussed in the manuscript 


\section{ABSTRACT}

32 Objectives: To describe and compare corneal peripheral angles in normal and keratoconic eyes, to gain a better understanding of the topography of the periphery of the cornea in keratoconus and assist practitioners in the selection and fitting of large diameter contact lenses.

Methods: Eighty-eight eyes were included in the study, divided in three groups: healthy (A0, 28 eyes), keratoconus at stage I according to the Amsler-Krumeich classification (AI, 33 eyes) and keratoconus at stages II to IV (AII, 27 eyes). The Pentacam Scheimpflug system was employed to manually measure the corneal peripheral angles corresponding to a chord length range between 8.6 and $12.6 \mathrm{~mm}$ at eight different peripheral locations.

Results: The peripheral angle was influenced by ocular condition and by the peripheral location, with no interaction effect between both factors. Statistically significant differences were found in mean corneal peripheral angles between group A0 $\left(30.84^{\circ} \pm 2.33^{\circ}\right)$ and AI $\left(31.63^{\circ} \pm 2.02\right)(\mathrm{p}=0.001)$ and between A0 and AII $\left(31.37^{\circ} \pm 2.11^{\circ}\right)$ $(\mathrm{p}=0.030)$. The differences between AI and AII were not significant. In all eyes, the

47 largest and smallest peripheral angles were found at the temporal inferior and temporal superior locations, respectively, with a mean difference between largest and smallest of $3.37^{\circ} \pm 1.42^{\circ}$ in healthy eyes and $2.96^{\circ} \pm 1.54^{\circ}$ in keratoconus (AI + AII).

Conclusion: Clinically insignificant differences were found in peripheral angles between keratoconus and healthy eyes, giving support to the use of large diameter, intralimbal contact lenses with peripheral designs, and resting on the same corneal region, as those fitted on normal corneas. 
54 KEYWORDS: Keratoconus; Peripheral corneal geometry; Scheimpflug imaging;

55 Pancorneal contact lenses; Peripheral corneal angle 
Keratoconus is an ectatic corneal disorder characterized by progressive stromal thinning and cone-like protrusion, which may lead to irregular astigmatism, myopia and severely affect vision. ${ }^{1}$ Although the most commonly cited prevalence rate of keratoconus is 54.5 per 100,000 population, ${ }^{2}$ published prevalence rates may range from 0.3 per 100,000 in Russia $^{3}$ to 2340 per 100,000 in Israel. ${ }^{4}$ Recent evidence recovered from the tear film suggests an inflammatory component to the pathogenesis of keratoconus. ${ }^{5,6}$ In addition, oxidative stress may play an important role in the development and progression of this condition. ${ }^{7}$ Structural and anatomical changes, such as, stromal thinning and posterior stress lines, suggest a possible change in the geometry of the posterior cornea, independent of anterior surface alterations. ${ }^{8}$

At present, there are different options for the management of keratoconus, depending on the stage of the condition and on the effect on vision, including surgical and nonsurgical approaches. Among nonsurgical management strategies, corneal gas-permeable contact lenses are available for keratoconic patients with unsatisfactory vision with glasses or conventional soft contact lenses. ${ }^{9}$ Alternatively, contact lens options include pancorneal (lenses that rest on the corneal periphery), corneoscleral (lenses that rest partly on the cornea and partly on the sclera), and scleral lenses (lenses that rest completely on the sclera). ${ }^{9-12}$ To fit these types of lenses practitioners need detailed knowledge of the corneal, limbal and anterior scleral shape, and rely on complex corneal topographical descriptors beyond central keratometry values. ${ }^{12-13}$ Although central corneal shape is well described in the literature, only a few research efforts have been devoted to the analysis of peripheral corneal and corneo-scleral junction geometry. For instance, a previous study introduced a new parameter to describe the forward elongation and 
measured the corneal-scleral tangential angle between $10 \mathrm{~mm}$ and $15 \mathrm{~mm}$ (limbal angle) and between $15 \mathrm{~mm}$ and $20 \mathrm{~mm}$ (scleral angle) in 96 normal eyes. ${ }^{12,15}$ These authors noted flatter radii at the nasal than temporal quadrants of the eye and observed a more pronounced scleral than limbal toricity. The same research group also suggested using tangent angles rather than curves when fitting scleral lenses. ${ }^{12}$ Similarly, Sorbara et al ${ }^{16}$ measured the sagittal depth of the cornea and the scleral angles along the chord corresponding to the horizontal visible iris diameter (HVID) and again at $15 \mathrm{~mm}$ in normal and keratoconic eyes. These authors observed differences between normal and keratoconic eyes in peripheral scleral angles measured at the HVID at the inferior and temporal locations only, whereupon they highlighted the relevance of assessing sagittal depth, together with inferior and temporal angles when fitting very large diameter contact lenses. $^{16}$

Although corneal topography is considered the most sensitive and commonly used method to detect keratoconus, ${ }^{1}$ tomography (e.g., Scheimpflug Imaging System) has been described as the best available current technique to diagnose early keratoconus. ${ }^{9}$ The Scheimpflug Imaging System captures information of the entire anterior segment from the anterior corneal surface to the lens, and assesses several corneal and anterior chamber parameters. ${ }^{17}$ This device, and others, such as, the Visante ${ }^{\mathrm{TM}}$ (Carl Zeiss, Dublin, CA) anterior segment optical coherence tomographer (OCT), provide images and/or software to derive, through the appropriate analysis, relevant information about the peripheral cornea. Thus, for instance, the Visante ${ }^{\mathrm{TM}}$ was used by van der Worp and co-workers and by Sorbara and colleagues to measure scleral and peripheral corneal angles. $^{12,15,16}$

With the aid of this instrumentation, new studies may be conducted to gain a better understanding of the geometry of the periphery of the cornea to aid in the design and 
106 fitting strategies of new contact lenses for keratoconic eyes, as either an alternative or 107 complementing the traditional approach based on corneal radii. With this purpose, the 108 present study employed Scheimpflug images to explore peripheral corneal angles in a 109 sample of keratoconic eyes, and compared them with a group of age-matched healthy 110 eyes. The area under study, corresponding to a chord length range between 8.6 and 12.6 $111 \mathrm{~mm}$, has not been previously investigated with this instrumentation, albeit this region 112 may be considered of relevance, as a resting area of large diameter intralimbal contact 113 lenses used both for keratoconus and healthy eyes. 


\section{METHODS}

\section{Study Sample}

Sixty eyes from 60 patients with keratoconus were included in the study. Patients were selected from those presenting for eye care or contact lens fitting. The same corneal specialist diagnosed and classified all the eyes with keratoconus according to the Amsler-Krumeich classification: ${ }^{18} 33$ eyes were at stage I, 18 eyes at stage II and 9 eyes at stage IV. In patients with bilateral keratoconus, only one eye was included in the study, selected at random. In addition, 28 healthy eyes from 28 patients were included in an age-matched control group. Eyes that had a history of ocular or refractive surgery, contact lens wear, ocular trauma or corneal pathology other than keratoconus were excluded from the study. All participants provided written informed consent after the nature of the study was explained to them. The study was conducted in accordance with the Declaration of Helsinki tenets of 1975 (as revised in Tokyo in 2004).

\section{Procedure}

The Pentacam Scheimpflug System (software version 1:18, Optikgeräte Oculus GmbH, Wetzlar, Germany), which was calibrated according to the recommendations of the manufacturer, was used to analyse the corneal and anterior segment parameters. All Pentacam measurements were conducted following the guidelines of the manufacturer. An experienced examiner captured three consecutive images from each eye.

The Pentacam software was used to determine the anterior best-fit-sphere (BFS), the anterior chamber depth from the corneal endothelium (ACD_end) and the anterior chamber volume (ACV). In addition, Scheimpflug images corresponding to the $180^{\circ}$ $0^{\circ}$ meridian were used for the determination of the length of the horizontal chord 
140 (LHC). Finally, the images corresponding to the $180^{\circ}-0^{\circ}, 45^{\circ}-225^{\circ}, 90^{\circ}-270^{\circ}$ and $141135^{\circ}-315^{\circ}$ meridians were retrieved to determine the angles of the corneal periphery at 142 both ends of each meridian. Therefore, eight angles were considered for each eye: 143 Superior (S), Nasal-Superior (NS), Nasal (N180), Nasal-Inferior (NI), Inferior (I), 144 Temporal-Inferior (TI), Temporal (T180) and Temporal-Superior (TS). The positions 145 corresponding to these angles are shown in Figure 1.

147 Corneal peripheral angles were measured manually on Scheimpflug images, using the tools of the freely available software Image J (Version 1.46a, Wayne Rasband, National Institute of Mental Health, Bethesda, Mariland, USA). Firstly, the Pentacam software option "Show Pixel Edge" was used to mark the areas where the cornea first lost transparency, whereupon the first pixels of these areas were employed as anchor points from which a straight line (chord) was traced joining both sides of the cornea (Figure

2). The length of this chord was measured in pixels and a segment $x$ was defined with a length equal to $10 \%$ of the total length of the chord. Finally, a $y$ segment was drawn forming a right angle from the end of $x$ to the corneal surface. The corresponding angle for that meridian $(\theta)$ was therefore obtained from $\arctan (y / x)$, that is, angles were measured with respect to the horizontal line. error, in which it was determined that this length resulted in the hypotenuse of the triangle defined by the catheti $x$ and $y$ to be tangent to the surface of the cornea. Thus, an increase in the length of $x$ to $15 \%$ of chord line was found to result in loss of tangency, whereas the relative magnitude of measurement error did not support using shorter lengths than the defined $10 \%$. All angle measurements were performed by the 
same trained technician, masked for the purpose of the study, who obtained three values (one for each of the recordings) from each eye. The angle value for any position and eye was calculated as the mean of these three values. Figure 3 and Figure 4 provide examples of small $\left(28.82^{\circ}\right)$ and large $\left(33.31^{\circ}\right)$ peripheral angles, as measured with this technique.

It must be noted that in some eyes the superior portion of the $90^{\circ}-270^{\circ}$ meridian was partly covered by the superior eyelid. In these cases, data from the superior angle were unavailable. To measure the inferior angle, a line was drawn starting at the pixel edge marking the loss of corneal transparency at $270^{\circ}$ and parallel to the anterior plane of the iris. By moving along this line, the length of $x$ was defined as the $20 \%$ of the distance between the point of loss of corneal transparency at $270^{\circ}$ and the geometrical centre of the pupil.

Once all visible angles were determined for each particular eye, the differences between the largest and smallest angles were calculated.

\section{Data analysis}

The statistical analysis of the data was conducted with the Minitab ${ }^{\circledR} 17$ Statistical Software. The data were first examined for normality with the Kolmogorov-Smirnov test, revealing the occurrence of a normal distribution. Consequently, the values of the corneal peripheral angles were submitted to a two-way analysis of variance (ANOVA), in which the considered independent variables or factors were ocular condition (healthy eyes [A0], keratoconic eyes at stage Amsler-Krumeich I [AI], keratoconic eyes at stages Amsler-Krumeich II and IV [AII]), and peripheral location (S, NS, N180, NI, I, TI, 
190 T180, TS). Whenever a statistically significant difference was found, post-hoc, pair-

191 wise comparisons were conducted with the Tukey's test. In addition, a t-test was used to 192 compare normal and keratoconic eyes (pooling together AI and AII) in terms of the 193 difference between the largest and smallest angles. Finally, the correlations between the 194 mean angle for each eye and the corresponding values of BFS, ACD_end, ACV and 195 ACD_end/ LHC were examined with a Pearson's coefficient of correlation test to 196 determine whether any of these anterior segment parameters was a good predictor of 197 peripheral corneal angle. In addition, the correlation between the measured chord 198 lengths and the corresponding angles was explored to investigate whether possible 199 differences in angles amongst the meridians originated in the actual peripheral location 200 under analysis. A p-value of 0.05 or less was defined as the cut-off point for statistical 201 significance. 
Sample demographics

Keratoconus patients were $34.4 \pm 14.5$ years old (mean \pm SD). Thirty-three eyes were (AII). Healthy patients (A0) were aged $32.8 \pm 13.7$ years. No statistically significant differences were revealed in neither age $(p=0.8)$ nor LHC $(p=0.62)$ between the three groups.

\section{Peripheral corneal angle analysis}

213 The mean and SD values of the corneal angles of each examined peripheral location for

214 healthy and keratoconic eyes are shown in Figure 5, using a right eye for data presentation. In less than $50 \%$ of the patients, data were available at the $\mathrm{S}$ position, which prevented statistical analysis being conducted for that particular peripheral location. When submitted to a two-way ANOVA test, statistically significant differences were found for each of the considered factors $(\mathrm{p}<0.001)$, that is, peripheral angle was influenced by ocular condition (A0, AI or AII) and by peripheral location. However, no interaction effect between both factors was revealed $(p=0.475)$. Table 1 presents a summary of the chord lengths for each of the meridians under consideration, classified by ocular condition. Overall, chord length ranged from between $8.6 \mathrm{~mm}$ to $12.6 \mathrm{~mm}$.

Regarding the factor ocular condition, Figure 6 displays a summary of the peripheral angle for each group (data are shown as mean and confidence intervals at 95\%). The Tukey test revealed statistically significant differences between A0 and AI ( $p=0.001)$ and between A0 and AII ( $\mathrm{p}=0.030)$, with smaller peripheral angles for the healthy 
group of patients. No statistically significant differences were found between AI and

230 AII $(\mathrm{p}=0.743)$. It must be noted, however, that even if statistically significant, these

231 findings were probably not clinically significant. As may be observed in Figure 7, which shows the frequency distribution of mean peripheral angles for healthy eyes and keratoconic eyes (groups I and II pooled together), there is a large overlap between healthy eyes and those with keratoconus, with an actual difference of only $0.69^{\circ}$ between the mean peripheral angle values.

The analysis of the influence of the factor peripheral location revealed that the largest and smallest mean peripheral angles were located at the TI $\left(32.03^{\circ} \pm 2.16^{\circ}\right)$ and $\mathrm{T} 180$ $\left(31.73^{\circ} \pm 2^{\circ}\right)$, and at the $\operatorname{TS}\left(30.26^{\circ} \pm 1.92^{\circ}\right)$ locations, respectively. Statistically significant differences were found between the mean peripheral angle values at TI and TS $(\mathrm{p}<0.001)$ and between T180 and TS $(\mathrm{p}=0.003)$. In addition, statistically significant differences were found between NI and TI $(\mathrm{p}=0.004)$ and between TS and $\mathrm{N} 180(\mathrm{p}=0.033)$

The mean peripheral angle values for each position and ocular condition are displayed in Figure 8. It may be observed that, irrespective of condition, the largest peripheral angles are always located at the inferior temporal quadrant (I, TI or T180), whereas the smallest angles are at TS. The mean difference between maximum and minimum peripheral angles was of $3.37^{\circ} \pm 1.42^{\circ}$ for healthy eyes and $2.96^{\circ} \pm 1.54^{\circ}$ for keratoconic eyes (AI and AII pooled together). This difference did not reach statistical significance $(p=0.226)$. 
255 Table 2 presents a summary of the analysis of correlation between the mean corneal 256 peripheral angles for healthy and keratoconic eyes (AI and AII pooled together) and 257 other anterior segment parameters (BFS, ACD_end, ACV and ACD_end/LHC). Weak 258 to moderate significant correlations were found between the mean corneal peripheral 259 angles and BFS $(r=0.44 ; p=0.019)$, ACD_end $(r=0.48 ; p=0.01)$ and ACV $(r=0.60$; $260 \mathrm{p}=0.001)$ for healthy eyes, and ACV $(\mathrm{r}=0.41 ; \mathrm{p}=0.001)$ for keratoconic eyes, that is, 261 none of the measured anterior segment parameters could be considered a good predictor 262 of peripheral angle.

263

264 Finally, in the analysis of the correlation between chord lengths and angles, a 265 statistically significant lack of correlation was found $(\mathrm{r}=0.32 ; \mathrm{p}<0.001)$, that is, 266 changes in the variable chord length only explained $10 \%$ of the variance encountered in 267 the measured angle (coefficient of determination of 0.105 ). 
271 Smaller peripheral corneal angles were found in healthy eyes than those reported by 272 both van der Worp and colleagues for their "limbal angle"12,15 and by Sorbara et al, on 273 the HVID chord. ${ }^{16}$ Thus, van der Worp et al and Sorbara et al documented angles at the 274 different peripheral locations ranging from $37.6^{\circ}$ to $39.3^{\circ}$ and $38^{\circ}$ to $39.6^{\circ}$, respectively, whereas the present findings ranged from $29.8^{\circ}$ to $31.9^{\circ}$. It must be noted, however, that the region under investigation in the present study corresponded to a chord length range from 8.6 to $12.6 \mathrm{~mm}$, that is, angles were measured at the corneal periphery, not in the limbus or sclera, as in those reports. Besides, although overall chord length varied from $8.6 \mathrm{~mm}$ to $12.6 \mathrm{~mm}$, mean values for each group of patients were in agreement with published literature. ${ }^{19}$ Furthermore, no correlation was found between chord lengths and peripheral corneal angles, that is, it may be assumed that small variations in chord length amongst the meridians under evaluation had a negligible influence on angle measurements. Finally, the employed methodology, in which we conducted all angle measurements considering a segment of $1 / 10^{\text {th }}$ of the chord length, ensured that all measurement points were at approximately the same relative distance from the limbus (with variations of the order of $0.1 \mathrm{~mm}$ ) for all patients and meridians. Interestingly, the measured angles and chord length range considered in this study are within the range of parameters commonly available in intralimbal lenses.

Regarding the factor ocular condition (A0, AI, AII), the present findings revealed a statistically significant change between A0 and AI but not between AI and AII, that is, whereas there is an increase in corneal peripheral angle at an early stage of keratoconus, this change does not seem to continue to later stages of the condition (Figures 6 and 7), 
albeit as the present sample of patients did not include enough keratoconus at stage III and IV this finding must be interpreted with caution. However, it must be noted that the differences in peripheral angles encountered in this study between normal and keratoconic eyes (AI+AII) were very small (Figure 7, mean difference of $0.69^{\circ}$ ) and probably clinically insignificant. In this regards, these findings are not dissimilar to those of Sorbara and colleagues, ${ }^{16}$ who did not observe any overall difference between keratoconus and healthy eyes, although these authors measured angles at the HVID chord diameter, used a different measuring technique and did not take into consideration differences in disease progression.

With reference to the factor peripheral location (NS, N180, NI, I, TI, T180, TS), the 305 largest and smallest mean corneal peripheral angles were found at the TI $\left(32.03^{\circ} \pm\right.$ $\left.2.16^{\circ}\right)$ and $\operatorname{TS}\left(30.26^{\circ} \pm 1.92^{\circ}\right)$ positions, respectively. Overall, the largest peripheral angles corresponded to the temporal quadrant (I, TI i T180) (Figure 8). Sorbara et al ${ }^{16}$ also observed a significant difference at the temporal quadrant $(\mathrm{p}<0.001)$ in peripheral angles at the HVID chord, but only in the keratoconus eyes. Our findings suggest that keratoconic eyes had larger angles than normal eyes at all peripheral locations, whereas Sorbara et al only found larger angles for the keratoconus group at the superior and inferior positions, reporting larger nasal and temporal angles in healthy eyes than in

313 keratoconic eyes. ${ }^{16}$ As noted above, any comparison with previous research, however, must be approached with caution, as the chord lengths under consideration, the instrumentation and the measurement technique (manual versus automatic) were not the same. 
318 The mean maximum differences in corneal peripheral angles in the present sample of 319 patients were of about $3^{\circ}$, both for keratoconus $\left(2.96^{\circ} \pm 1.54^{\circ}\right)$ and healthy eyes $\left(3.37^{\circ} \pm\right.$ $\left.1.42^{\circ}\right)$. In healthy eyes, this difference is either considered negligible when fitting revolution of symmetry large diameter intralimbal contact lenses (overall diameter of approximately $90 \%$ of HVID), or it is overcome using lens designs with a toric peripheral geometry, which rest on this area of the peripheral cornea. In keratoconic patients, the present findings give support to the use of large diameter intralimbal contact lenses, such as the Dyna IntraLimbal lens (Lens Dynamics, Golden, Colorado, US) or the Rose K2 IC lens (Menicon Co., Nagoya, Japan), which have a similar peripheral design to those employed in healthy eyes. Besides, it may be worth noting that small differences in peripheral angles may have an impact on edge lift, lens centration and stability, similar to healthy corneas. Thus, considering an ideal edge lift of $200 \mu \mathrm{m}$ and a landing zone width of $1 \mathrm{~mm}$, a change of $1^{\circ}$ in peripheral angle corresponds to approximately a $15 \mu \mathrm{m}$ change in edge lift, that is, up to $45 \mu \mathrm{m}$ changes in edge lift may be expected between peripheral quadrants (in keratoconic eyes the largest and smallest angles were always located at the same meridians). In these cases, a contact lens with a quadrant-specific peripheral design may be employed, a solution that is currently available for many lens designs, in particular for pancorneal ${ }^{10}$ and scleral

336 lenses. $^{20}$ Finally, our results suggest that lenses with peripheral conic designs may also be safely fitted in patients with keratoconus.

Correlation analysis revealed statistically significant associations between corneal 340 peripheral angle values and those of other anterior segment parameters, with the expected direction of the slope of the regression line and with the strongest correlation corresponding to ACV for both healthy and keratoconic eyes. However, all values of the 
coefficient of determination were relatively small, thus denoting that these anterior segment parameters are not good predictors of peripheral corneal angle and vice versa, and giving support to the need for direct measurement of peripheral angles on the Scheimpflug images to fully describe corneal periphery. ${ }^{16}$

It may be worth noting that the Pentacam System is not an ideal instrument to examine limbal angles. Indeed, given the characteristics of Scheimpflug images, measured angles with the Pentacam reside in the corneal periphery away from the limbus, at an area of interest when considering the peripheral geometry of large diameter intralimbal contact lenses. Besides, the same technique was employed to compare healthy and keratoconus eyes and, within the same group, to compare amongst the diverse meridians under examination. Therefore, any error in the present methodology would have a similar impact on all measurements, that is, comparison and relative values remain valid. Future research shall use anterior optical coherence tomography (OCT) technology to further explore these findings.

In conclusion, at a chord length between 8.6 and $12.6 \mathrm{~mm}$ the characteristics of the measured corneal peripheral angles were similar in healthy and keratoconus eyes. Indeed, the absolute values of peripheral angles and the difference between the largest and smallest angles were similar, irrespective of the condition, and within the normal range of peripheral parameters (tangential angle, overall diameter and angle selection steps) commonly found in large diameter intralimbal lens designs, albeit some keratoconus patients may benefit from toric designs or small quadrant-by-quadrant adjustments. The findings of this research may assist practitioners and lens manufacturers in the selection of peripheral lens designs for these patients. 


\section{REFERENCES}

370 1. Rabinowitz YS. Keratoconus. Surv Ophthalmol 1998;42:297-319.

2. Kennedy RH, Bourne WM, Dyer JA. A 48-year clinical and epidemiologic study of keratoconus. Am J Ophthalmol 1986;101:267-273.

3. Gorskova EN, Sevost'ianov EN. Epidemiology of keratoconus in the Urals. Vestn Oftalmol 1998;114:38-40.

4. Millodot M, Sheneor E, Albou S, et al. Prevalence and associated factors of keratoconus in Jerusalem: a cross-sectional study. Ophthalmic Epidemiol 2011;18:9197.

5. Jun AS, Cope L, Speck C, et al. Subnormal cytokine profile in the tear fluid of keratoconus patients. PLoS One 2011;6:e16437.

6. Lema I, Sobrino T, Durán JA, et al. Subclinical keratoconus and inflammatory molecules from tears. Br J Ophthalmol 2009;93:820-824.

7. Wojcik KA, Kaminska A, Blasiak J, et al. Oxidative stress in the pathogenesis of keratoconus and Fuchs endothelial corneal dystrophy. Int J Mol Sci 2013;14:1929419308.

8. Piñero DP, Nieto JC, Lopez-Miquel A. Characterization of corneal structure in keratoconus. J Cataract Refract Surg 2012;38:2167-2183.

9. Gomes JA, Tan D, Rapuano CJ, et al. Global consensus on keratoconus and ectatic diseases. Cornea 2015;34:359-369.

10. Kamar S, Vervaet C, Luyten GP, et al. Pancorneal contact lens with a toric edge: a new concept in keratoconus. Eur J Ophthalmol 2011;21:685-690.

11. Ramdas WD, Vervaet CJ. Progression of keratoconus in patients wearing 2014;37:251-256. 
12. van der Worp E, Bornman D, Ferreira DL, et al. Modern scleral contact lenses: A review. Cont Lens Anterior Eye 2014;37:240-250. rigid gas-permeable contact lens fitting in patients with keratoconus, and differences in age and gender. Cont Lens Anterior Eye 2014;37:20-25.

14. Mas-Aixala E, Gispets J, Lupón N, et al. The variability of corneal and anterior segment parameters in keratoconus. Cont Lens Anterior Eye 2016; 39:466-470. Spectrum 2010;6:26-32.

16. Sorbara L, Maram J, Mueller K. Use of the Visante ${ }^{\mathrm{TM}} \mathrm{OCT}$ to measure the sagittal depth and scleral shape of keratoconus compared to normal corneae: Pilot study. $J$ Optom 2013;06:141-146.

17. Kovács I, Miháltz K, Németh J, et al. Anterior chamber characteristics of keratoconus assessed by rotating Scheimpflug imaging. J Cataract Refract Surg 2010;36:1101-1106.

18. Krumeich JH, Kezirian GM. Circular keratotomy to reduce astigmatism and 410 improve vision in stage I and II keratoconus. J Refract Surg 2009;25:357-365. 19. Rüfer F, Schröder A, Erb C. White-to-white corneal diameter: normal values in healthy humans obtained with the Orbscan II topography system. Cornea 2005;24: 259261.

414 20. Van der Worp E. A Guide to Scleral Lens Fitting, second ed. Forest Grove, OR: 415 Pacific University; 2015. 
417 TABLES

418 Table 1. Summary of chord lengths for each of the meridians under consideration 419 (results are presented as mean \pm SD).

420

421

\begin{tabular}{ccccc}
\hline $\begin{array}{c}\text { Ocular } \\
\text { condition }\end{array}$ & \multicolumn{4}{c}{ Chord length (mm) } \\
\hline & $\mathbf{0 - 1 8 0}^{\circ}$ & $\mathbf{4 5 - 2 5 5}^{\circ}$ & $\mathbf{9 0 - 2 7 0}$ & $\mathbf{1 3 5 - 3 1 5}^{\circ}$ \\
Healthy & $11.61 \pm 0.36$ & $11.04 \pm 0.41$ & $10.19 \pm 0.84$ & $11.12 \pm 0.47$ \\
$\begin{array}{c}\text { Keratoconus } \\
\text { Stage I }\end{array}$ & $12.09 \pm 0.68$ & $11.45 \pm 0.60$ & $9.95 \pm 1.31$ & $11.53 \pm 0.51$ \\
$\begin{array}{c}\text { Keratoconus } \\
\text { Stages II-IV }\end{array}$ & $11.68 \pm 0.56$ & $11.03 \pm 0.44$ & $10.20 \pm 1.05$ & $10.89 \pm 0.62$ \\
\hline
\end{tabular}


423 Table 2. Correlation between the mean values of the corneal peripheral angles and the

424 other anterior corneal parameters under study (keratoconus eyes include AI and AII 425 groups).

426

\begin{tabular}{|c|c|c|c|c|c|c|}
\hline \multirow[t]{2}{*}{ Parameter } & \multicolumn{3}{|c|}{$\begin{array}{c}\text { Mean peripheral angle } \\
\text { (healthy eyes) }\end{array}$} & \multicolumn{3}{|c|}{$\begin{array}{c}\text { Mean peripheral angle } \\
\text { (keratoconic eyes) }\end{array}$} \\
\hline & $\overline{R^{2}}$ & Slope & $p$-value $*$ & $R^{2}$ & Slope & $p$-value $*$ \\
\hline BFS & 0.19 & 0.07 & 0.019 & 0.003 & 0.02 & 0.685 \\
\hline ACD_end & 0.229 & 0.1 & 0.01 & 0.059 & 0.07 & 0.062 \\
\hline $\mathrm{ACV}$ & 0.355 & 12.56 & 0.001 & 0.165 & 10.53 & 0.001 \\
\hline ACD_end/LHC & 0.124 & 0.006 & 0.066 & 0.02 & 0.177 & 0.279 \\
\hline \multicolumn{7}{|c|}{$\begin{array}{l}\text { or chamber depth measured from the endothelium } \\
\text { amber volume } \\
\text { he horizontal chord }\end{array}$} \\
\hline
\end{tabular}


435 FIGURES

436 Figure 1. Positions of the examined peripheral corneal angles for the right (RE) and left 437 (LE) eyes.

438

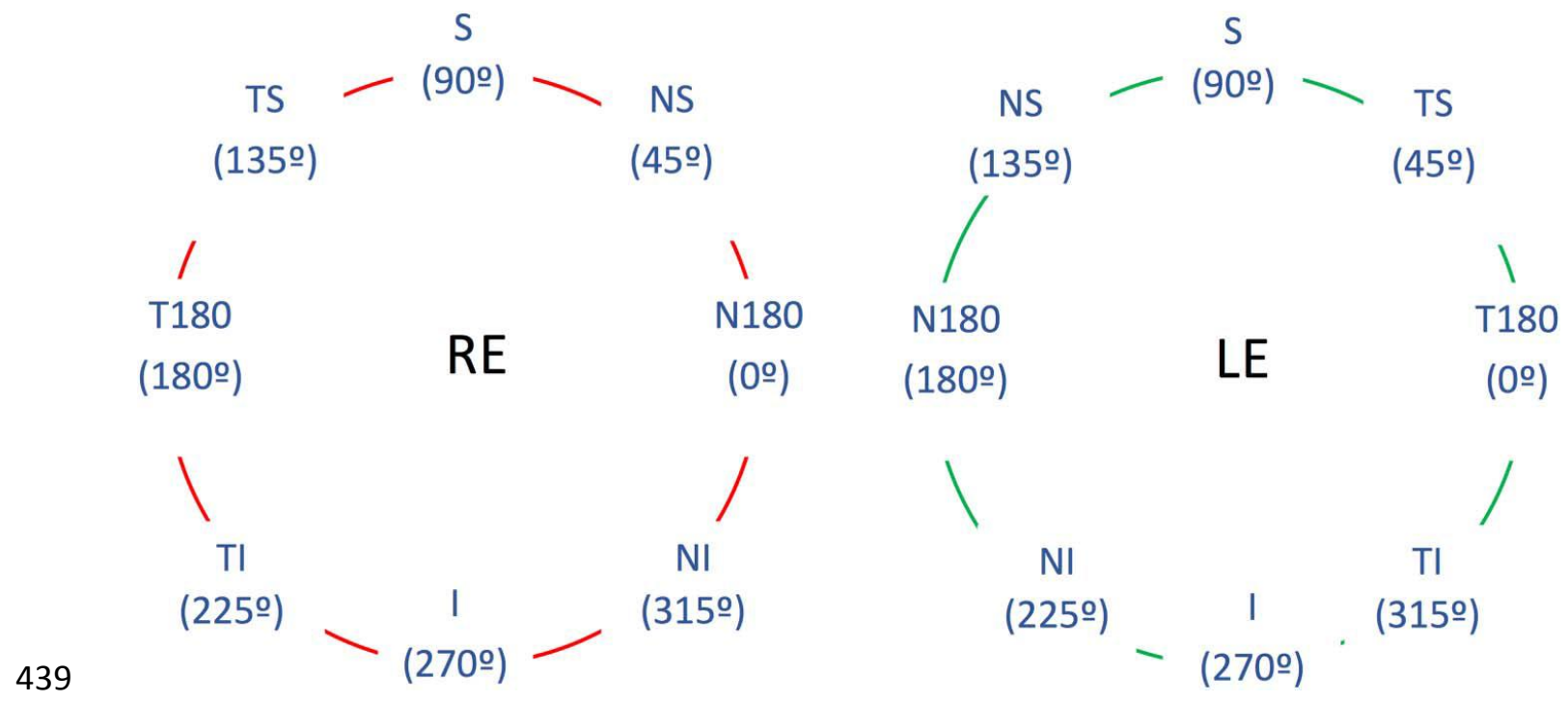

440 
441 Figure 2. Peripheral corneal angles measurement in a patient with keratoconus. [ $x$ : a

442 segment corresponding to $10 \%$ of the cord length joining the areas of loss of corneal 443 transparency; $y$ : a segment drawn perpendicularly from the end of $x$ to the anterior 444 surface of the cornea; $\theta$ : angle determined by $\theta=\arctan (y / x)]$.

445

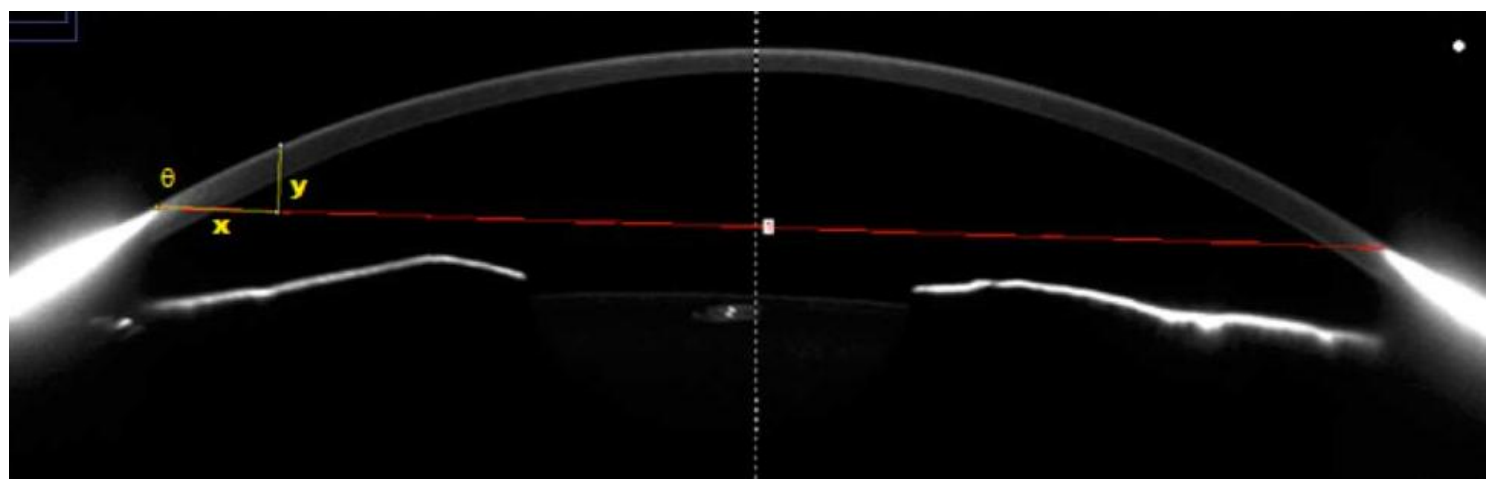

446

447 
448 Figure 3. Scheimpflug image showing the measurement of a small peripheral angle $449\left(28.82^{\circ}\right)$.

450

451

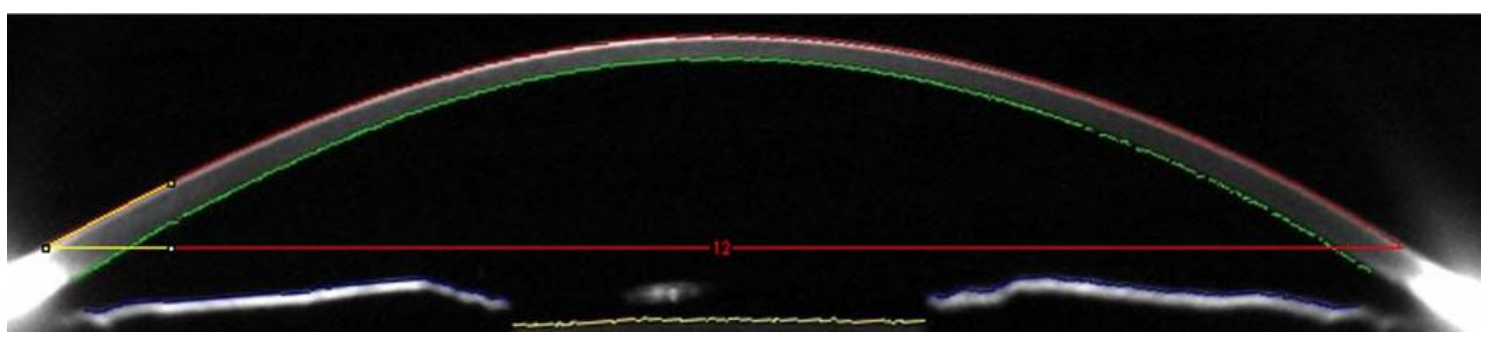

452 
453 Figure 4. Scheimpflug image showing the measurement of a large peripheral angle

$454\left(33.31^{\circ}\right)$.

455

456

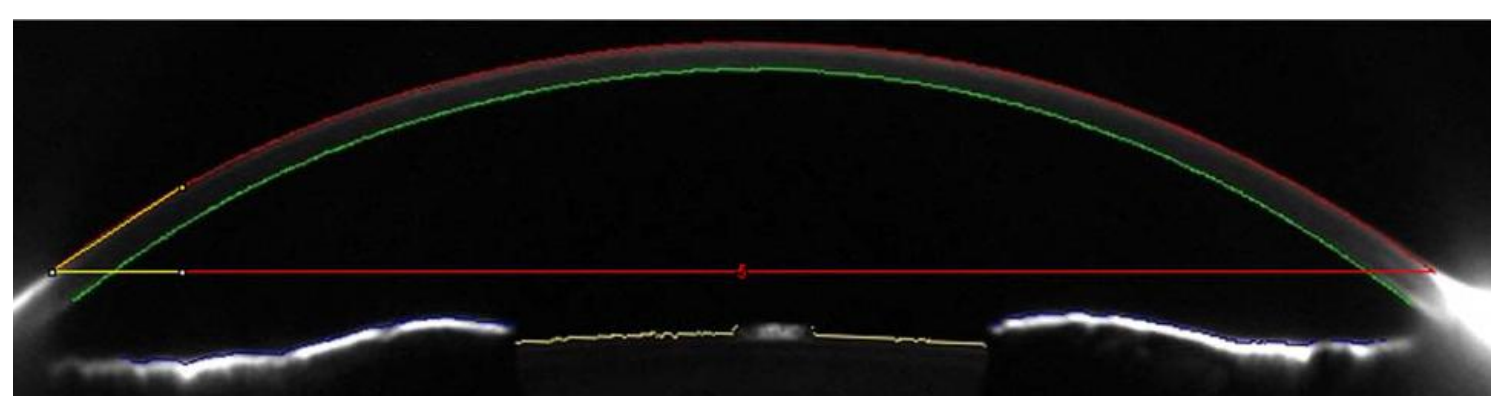

457 
458 Figure 5. Corneal peripheral angles in mean \pm SD in healthy (A0) and keratoconic eyes 459 of stage I (AI) and stages II and IV (AII). Data are displayed on a right eye.

460

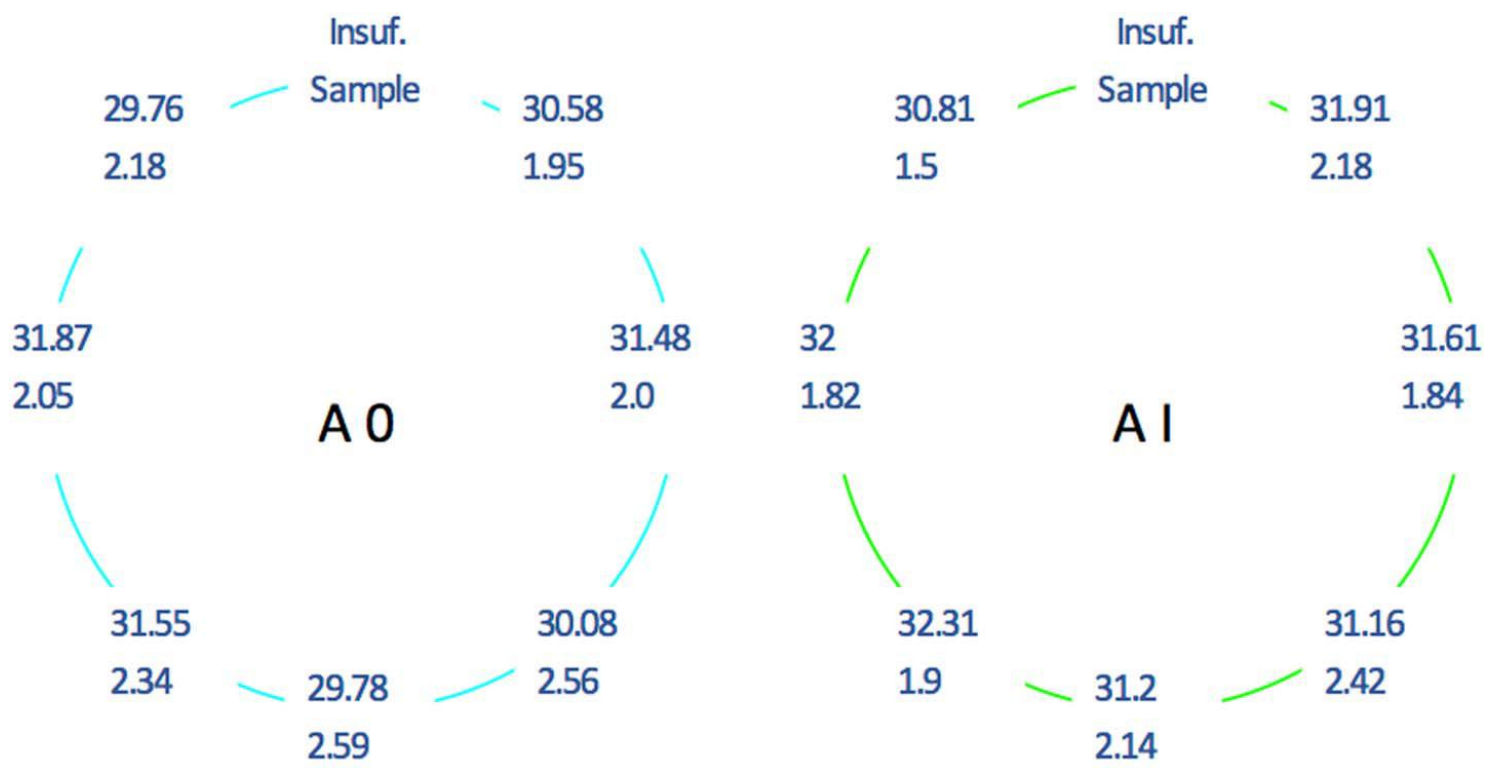

Insuf.
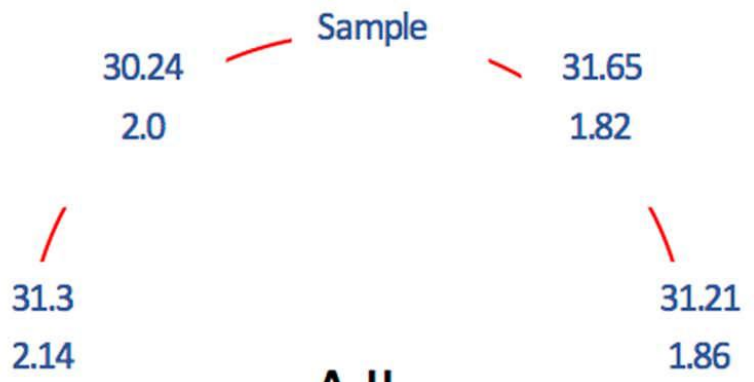

461

A II
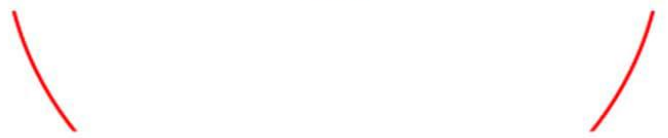

32.27

31.08

2.34

1.99

462 
463 Figure 6. Mean corneal peripheral angles in healthy (A0) and keratoconic eyes of stage 464 I (AI) and stages II and IV (AII). Error bars show confidence intervals at 95\%.

465

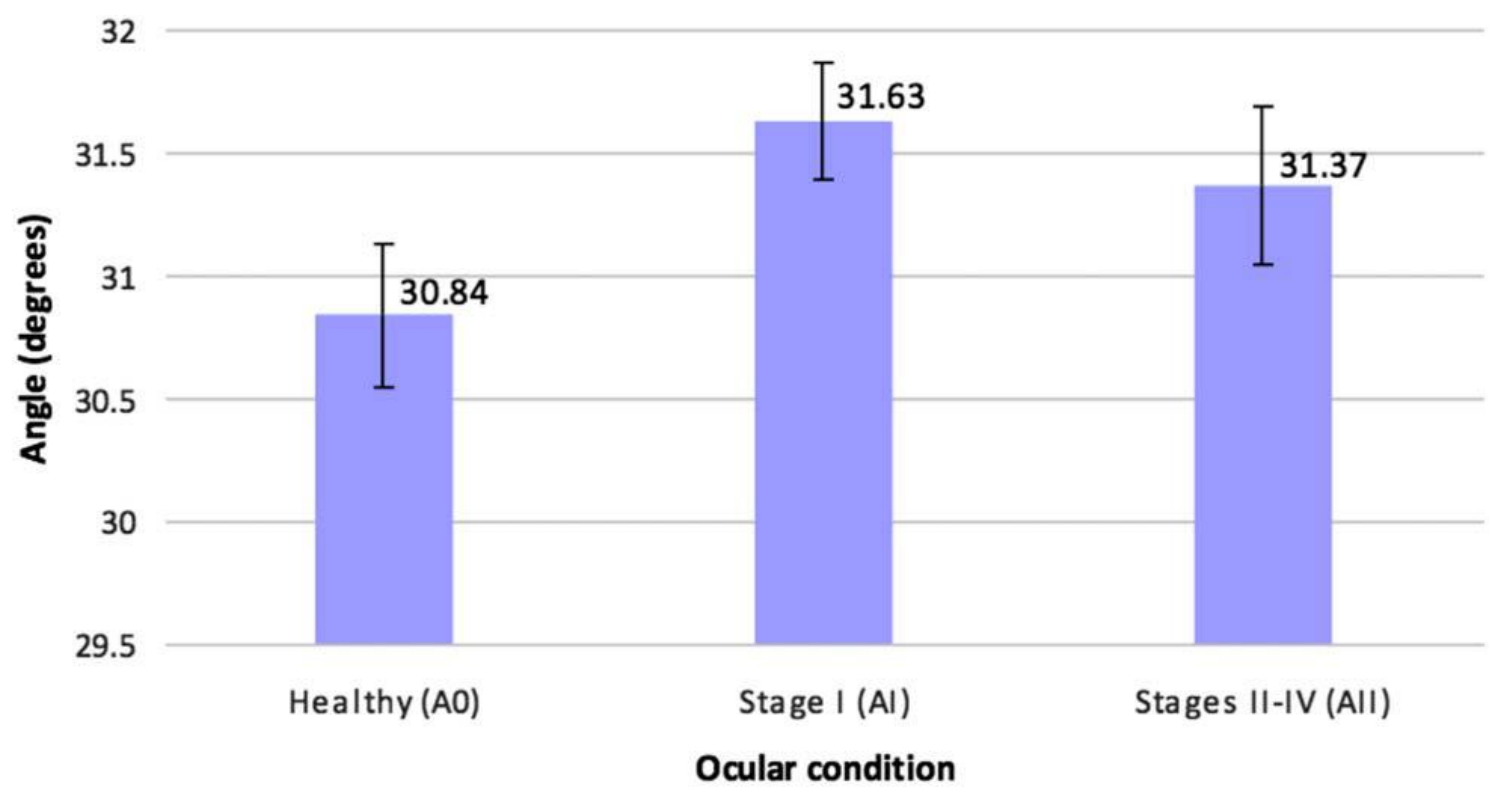

466

467 
Figure 7. Distribution of corneal peripheral angles in healthy (A0) and keratoconic eyes

469 (pooled data of AI and AII).

470

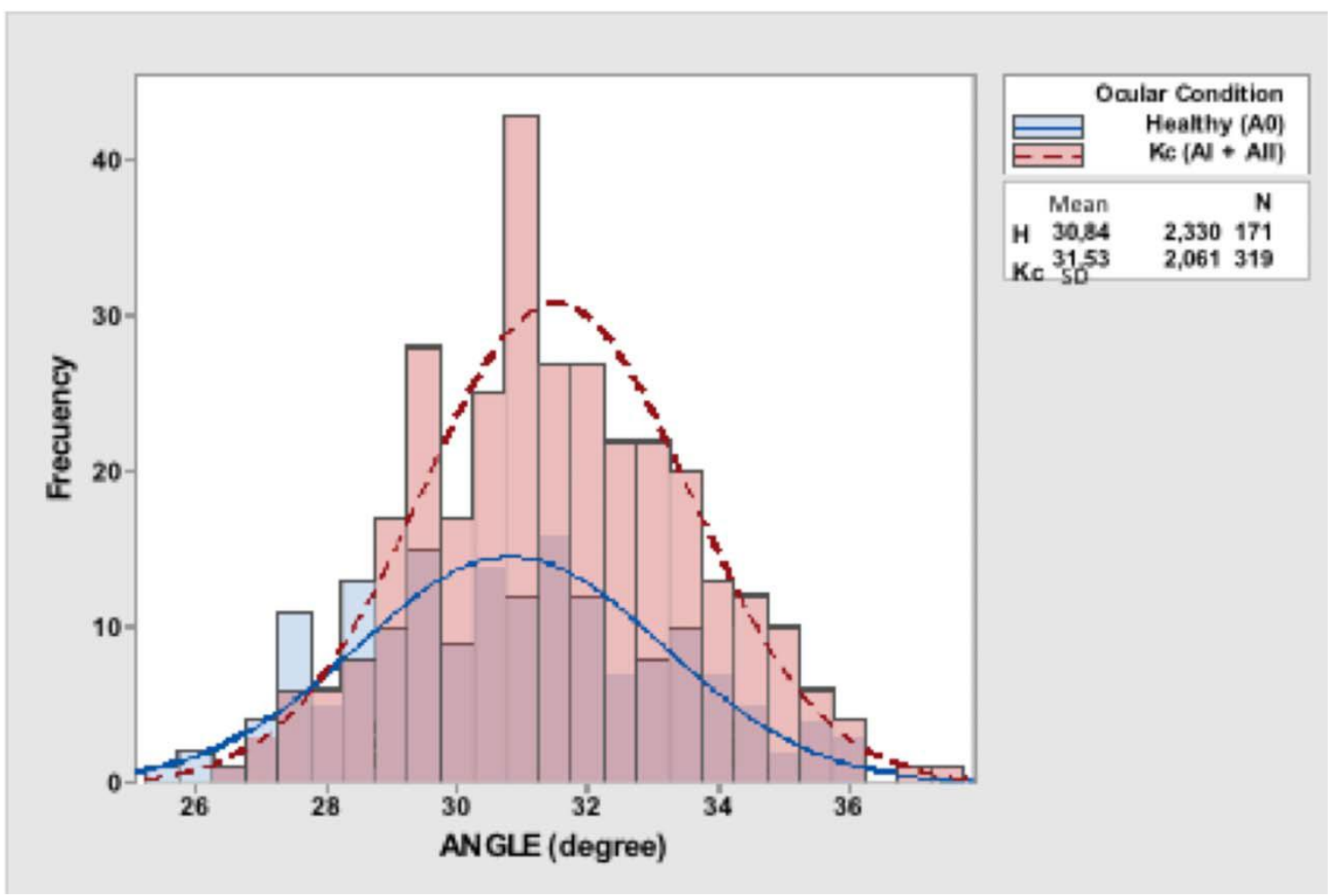

471

472 
473 Figure 8. Mean corneal peripheral angle values according to peripheral location (NS,

474 N180, NI, I, TI, T180, TS) and ocular condition (healthy [A0], keratoconic eyes stage I $475[\mathrm{AI}]$ and keratoconic eyes stages II-IV [AII]).

476

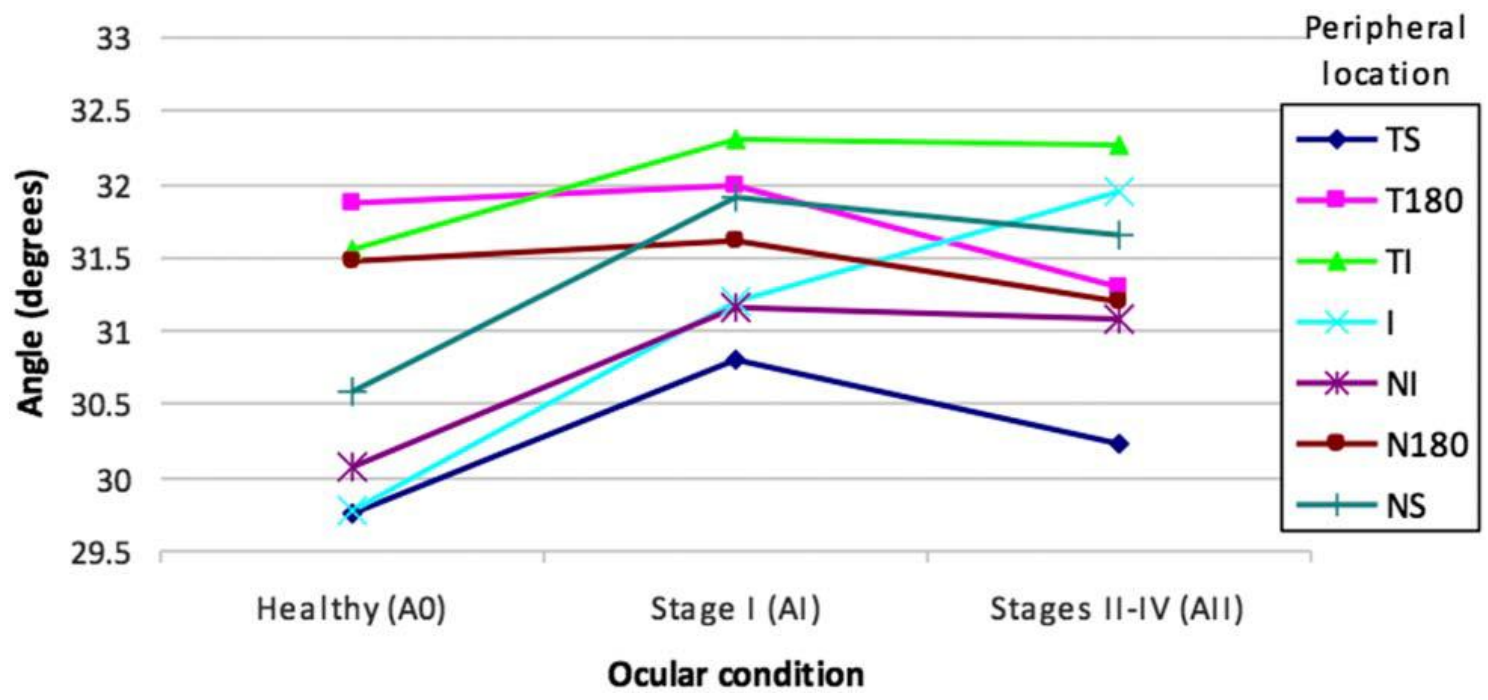

